\title{
Neurobiological Fingerprints of Psychiatric Disorders: An Opportunity to Refine Diagnostic Categorization
}

\author{
Rory Fleming Richardson* \\ Department of Clinical Medical Psychologist \& Neuropsychologist, Malaysia
}

*Corresponding author: Rory Fleming Richardson, Clinical Medical Psychologist \& Neuropsychologist, Clinical Research Malaysia, Ministry of Health, Malaysia.
Received Date: June 20, 2019

Published Date: July 01, 2019

\section{Short Communication}

Some of the problems which have plagued psychiatry and psychology relate to the limitations of our ability to research conditions to any extent that there is a preponderance of connective data to clarify specific differences in conditions. Part of the problem in achieving the best quality of research is that all life functions on a continuum, spanning time and changing over that medium. Most physical medical scientists know that gathering samples of blood, or blood pressure, or other measures must be repeated at the same time and under the same conditions every time to be able to make accurate comparisons. Blood pressure in the morning will be different from the blood pressure at night [1]. Blood chemistry after fasting all night is different than blood chemistry three hours after dinner. Almost all of medical science is an act of archeology, gathering samples of a point in time, and understanding that they are small representations of one point in time which by the time they are taken have already changed [2]. Attempting to accurately sample either the minutia or Gestalt of physiology along a timeline that takes in months is beyond what most research can achieve without prohibitive costs. Thus, we find ourselves reading research which provides data that may not include when the data was gathered or reference to the possibility that the data may alter with the variable of "time."

When we consider that something as slight as a smile from the research assistant can alter the neurochemistry and neurophysical state of a subject, we find that our research data on emotional states may be subject to errors found in early comparative psychology, such as Clever Hans Phenomenon [3]. Gathering enough of a neurobiological fingerprint of a condition which can provide the continuum of process, factoring in time, is difficult. Add in the subtle factors of experimenter/subject interaction further complicating the picture without a clear protocol. If we add in the unique differences between the subjects belief systems and their impact on physiological response, research starts to appear overwhelming [4]. Despite these potential complicating variables, we have been able to gather significant clues as to neurophysiological differences of different psychiatric disorders.

With that said, there is another problem which plagues clinical practice of diagnostics. The Diagnostic and Statistical Manual (DSM) used today evolved from the works of Emil Kraepelin. Over the years, it evolved from the "psychosis/neurosis" era to include many more categories and refined detail [5]. The DSM eventually moved to basic categorization of Axis I and Axis II diagnoses. Originally, back in the developmental stages of the DSM-III, personality disorders were learned, adaptive behaviors and thinking patterns which developed based on the individuals exposure to environment early life. Thus, the use of personality disorders were reserved for later adolesence and adults. Throughout the "decade of the brain" to current times, the neurobiology of personality disorders have been studied. We do make changes to the physiology of our brains through continual synaptic remodeling, and the impact of environmental nutrients and toxins. For individuals who have undergone trauma, there is evidence supporting changes in not only brain physiology, but actual changes in the nodes of the amygdala and related areas. So, if there is a biological fingerprint that spans generations, is it an Axis I or Axis II diagnosis?

Should the definition and criteria for Borderline Personality Disorders (BPD), which appears to have genetic indicators, be changed into one or more new different diagnoses. The overlay between affective disorders (including bipolarity) have been explored. There is evidence that suggests an overlap between Borderline Personality Disorders and affective disorders [6,7]. I have seen several bipolar patients with atypical, but definite, 
cyclic patterns. Some go from a significant depressive phase to a profound, dark, almost violently agitated depressive phase. Some have extremely short cyclic patterns lasting a day. The current limitations of the types of bipolarity forces clinicians into an Atypical or Unspecified category which does not serve well for refinement of diagnostic research. There are also concomitant disorders with Borderline Personality Disorder, complicating neuroimaging and researching neurophysiological elements. Does this suggest overlaying conditions, or the possibility of different conditions, within what we have labeled Borderline Personality Disorder? The criteria for BPD is also relatively vague. The DSM-V took a significant leap in improving the criteria for this diagnosis which, if one knows how to use, can help sort out the symptoms of other disorders and situations. But would it not be better to separate the different criteria into specifiers which can be used to clarify categories for research and treatment? Would it not be better to clearly describe the pattern of behavior in more detail? The "you are a saint/you are the devil" pattern is one. Being crisis addicted is another. Not being able to maintain a close relationship is another. This is one of the reasons we need to consider using neurobiological fingerprints as a way of refining our diagnostic coding, so that we can match behavioral symptoms with the biology. In treating BPD, some respond to SSRI's, some respond to atypical antipsychotics or mood stabilizers, while others respond to other medications [6]. If we were able to further clarify these, and identify the ones which are possibly genetic, we could find ourselves with a new diagnoses which falls into one of the other Axis I spectrums. One possible diagnosis would be for individuals presenting a cyclic mood cycle with a genetic indicator supporting a mood disturbance, with all the behavioral patterns of borderline personality disorder. If there is a family history of a rapid cyclic mood disturbance with borderline personality features which is most responsive to mood stabilizers, naming this condition Borderline Bipolar Disorder may be not only descriptive, but also provide a clearer treatment direction including psychotherapeutic and psychopharmacological intervention. With the use of universally accepted neuroimaging protocol, extended family history and identification of borderline criteria, standards could be establish to determine the diagnosis.

Daniel Amen, M.D. was the principle pioneer in the application of SPECT to identify neurophysiological/neurophysiological patterns seen in psychiatric conditions, and their comparative similarities and differences. He was profoundly riticuled by both professionals attempting to hold to the old paradigm, as well as those who simply did not have the educational background to understand his work in comparison to the neurobiological landscape of current psychiatric/neuropsychological knowledge. The patterns that he found between Obsessive Compulsive Disorder and Oppositional Defiant Disorder are but one of the examples. One of the greatest strengths in his research is that a very specific protocol, and use of specific imaging equipment, is used each time. The problem with the research being done using PET and SPECT by various researchers is that they simply do not use the same protocol [7]. Given the number of scans Dr. Amen has (over 100,000 scans), perhaps his protocol and type of imaging unit should be one of the "standard."
From the days of Wilder Penfield to Donald Hebb, we have been attempting to capture the illusive engram and other patterns, which are momentary points in time; which when discovered rivals quantum physics. Neural connections remodel and change throughout the brain creating new pathways within the milieu of the brain's topobiology [8]. Neurochemicals and metabolites move, changing the landscape known as neurophysiology. The Gestalt of the entire body alters and interacts with all of these changes and more as we each pass through the moments of time. If we include the laws of quantum entanglement and quantum physics, our minds would explode with the variables. Why did I become a clinical biopsychologist/medical psychologist? It is the same reason that we travel into the unexplored depths of the sea or the vastness of space. In hope that the areas we have not explored will help others and make the world a better place. For me, the exploration of the human psyche of each individual is the frontier, and the art in the blend of known science to help these individuals on a path of alchemy changing negative experiences, diseases, and problems, into strengths and beauty is exciting. Unfortunately, many psychologists have elected to stay in a more limited scope: electing not to learn more about anatomy, physiology, biochemistry, cytology, microbiology, and the dynamic interplay of life within/ between the mind and the body. Each elected subset of the field is of value. It is extremely hard to see the subtle ballet of life if you do not understand the physiology of human existence nor are able to understand the complexity hidden in various research. Something as basic as taking a thorough developmental history is potentially one of the best diagnostic sources. Within the developmental history, the clinician can find out about: (1) the environment the patient experienced, (2) behavioral information about parents and siblings which can help determine potential genetic predisposition as well as learned behaviors, (3) onset of symptoms, (4) variations between siblings which can suggest commonalities of genetics and biology, (5) variations based on birth order, adaptive functioning, and other variants, and (6) impact of developmental delays and physical illnesses. There are times that we are all too willing to accept the genetic links for physical illnesses but dismiss the genetic and biological links of psychiatric conditions. If a clinician is not versed in the medical sciences, clues can be missed or misinterpreted.

One of the challenging realities in psychiatry and psychology is that patients frequently come in with more than one issue. The comorbidity of anxiety and depression complicates our ability to research these conditions. It is not unusual for individuals with Obsessive Compulsive Disorder, after being dragged around by the haunting obsessions and compulsions for years, to become profoundly depressed. I frequently have seen patients who have been in treatment for years for depression and/or anxiety left with little hope of any improvement. In many cases, I will administer the Yale-Brown Obsessive Compulsive Checklist and Scale, only to find that they suffer from Obsessive Compulsive Disorder. The question arises "which came first?" Ferreting out the dance and interplay of biology, social interaction, personal response and choice, and belief system is far from easy, but it is possible with experience, time, and effort. 
Various psychotherapy approaches including dialectical behavior therapy, 12-Step recovery (Emotions Anonymous), and others have proven valuable. Yet when we start to see medications such as depakote or atypical antipsychotics, significantly helping the functionality of individuals diagnosed with Borderline Personality Disorder, we need to consider that there is an alternative form of a bipolar condition which has yet to be named. After muddling around the psyche of thousands of individuals, as well as your own for half a century, you become extremely comfortable with the fluid milieu of biopsychosociospiritual existence. You start to see patterns of biology, genetic, environment, thought, and belief, which provide the Gestalt of each human being. The time for the inclusion of neurophysiology and neuroscience in the development of diagnostic categories has arrived.

\section{Acknowledgement}

None.

\section{Conflict of Interest}

No conflict of interest.

\section{References}

1. Morris CJ, Hastings JA, Boyd K, Krainski F, Perhonen MA, et al. (2013) Day/night variability in blood pressure: influence of posture and physical activity. Am J Hypertens 26(6): 822-828.

2. Reddy S, Sharma S (2019) Physiology, Circadian Rhythm. [Updated 2018 Oct 27]. In: Stat Pearls [Internet]. Treasure Island (FL): Stat Pearls.

3. Samhita L, Gross HJ (2013) The "Clever Hans Phenomenon" revisited. Communicative \& integrative biology 6(6): e27122.

4. Persinger M (2001) Neuropsychiatric Practice and Opinion. The Neuropsychiatry of Paranormal Experiences 13(4): 515-524.

5. Shorter E (2015) The history of nosology and the rise of the Diagnostic and Statistical Manual of Mental Disorders. Dialogues in clinical neuroscience 17(1): 59-67.

6. Witt SH, Streit F, Jungkunz M, Frank J, Awasthi S, et al. (2017) Genomewide association study of borderline personality disorder reveals genetic overlap with bipolar disorder, major depression and schizophrenia. Transl Psychiatry 7(6): e1155.

7. Daniel G Amen, Manuel Trujillo, Andrew Newberg, Kristen Willeumier, Robert Tarzwell, et al. (2011) Brain SPECT Imaging in Complex Psychiatric Cases: An Evidence-Based, Underutilized Tool. The open neuroimaging journal 5: 40-48.

8. Edelman GM, Gally JA, Baars BJ (2011) Biology of consciousness. Frontiers in psychology 2: 4. 